\title{
Unanticipated prognosis of differential thyroid cancer patients with TO stage: analysis of the SEER database 2004-2013
}

\author{
Chunping Liu ${ }^{1, *}$, Jie Ming ${ }^{1, *}$, Wen Zeng ${ }^{2}$, Shuntao Wang ${ }^{1}$, Yiquan Xiong ${ }^{1}$, Qiuyang \\ Zhao $^{1}$, Xingjie Yin ${ }^{1}$, Zeming Liu ${ }^{1}$ and Tao Huang ${ }^{1}$ \\ ${ }^{1}$ Department of Breast and Thyroid Surgery, Union Hospital, Tongji Medical College, Huazhong University of Science and \\ Technology, Wuhan 430022, China \\ ${ }^{2}$ Department of Ophthalmology, Zhongnan Hospital, Wuhan University, Wuhan, China \\ *These authors have contributed equally to this work \\ Correspondence to: Tao Huang, email: huangtaowh@163.com
}

Zeming Liv, email: 6myt@163.com

Keywords: differential thyroid cancer, TO stage, prognosis, SEER

Received: May 23, $2017 \quad$ Accepted: July 13, $2017 \quad$ Published: August 07, 2017

Copyright: Liu et al. This is an open-access article distributed under the terms of the Creative Commons Attribution License 3.0 (CC BY 3.0), which permits unrestricted use, distribution, and reproduction in any medium, provided the original author and source are credited.

\section{ABSTRACT}

The prognosis of T0 stage differentiated thyroid cancer (DTC) remains unclear. This study aimed to investigate the prognosis of TO stage DTC patients to provide a new perspective on treatment guidelines for these patients. We investigated a large cohort of DTC patients from the Surveillance, Epidemiology, and End Results (SEER) database between 2004 and 2013. Patient survival curves were examined by Kaplan-Meier analyses with log-rank tests and Cox proportional hazards regression analyses. In the study cohort, the rate of cancer-specific mortality per 1000 personyears for T0 was higher than T1-T3, but lower than T4. The all-cause mortality for T0 patients was higher than all other stages (T1-T4). Multivariate Cox regression modeling showed that TO had a significant risk for cancer-specific mortality when compared to $\mathrm{T} 1$ and $\mathrm{T} 4$, but not $\mathrm{T} 2$ or T3, after adjustment for other risk factors. For all-cause mortality, T0 showed a significant risk for all-cause mortality when compared to T4, but not T1-T3 stage patients. Similar results were obtained after matching for influential factors using propensity scored matching analysis. The unanticipated prognosis of TO stage DTC patients was found to be not better than of other stage DTC patients, providing new implications for the treatment of TO stage DTC patients.

\section{INTRODUCTION}

The incidence of thyroid cancer has risen rapidly in recent decades [1-4]. The follicular cells give rise to two main forms of differentiated thyroid cancer (DTC): papillary thyroid carcinoma and follicular thyroid carcinoma. DTC accounts for approximately $86 \%$ of all thyroid carcinomas $[1,5]$.

The current American Joint Committee on Cancer (AJCC) staging systems: TNM system (versions 6.0 and 7.0) are prognostic systems that predict DTCspecific mortality [6]. These systems are considered gold standards to stratify cancer patients into different risk groups. T0 patients are defined as not having evidence of a primary tumor, according to the TNM system, in both versions 6 and 7. A diagnosis of T0 stage DTC would be confirmed by Fine Needle Aspiration (FNA) biopsy (histological and cytological confirmation) or radioactive iodine uptake test in metastasis sites such as the brain, bone, and lung [7].

The Surveillance, Epidemiology, and End Results (SEER) program of the National Cancer Institute (NCI) is the largest publicly available source of data on cancer incidence and survival $[8,9]$. There are few 
investigations focusing on the prognosis and treatment of T0 stage DTC patients. In a previous study, T0 patients were considered to have a low mortality and had less aggressive treatment as compared to $\mathrm{T} 1-\mathrm{T} 3$ patients [10]. In the present study, we evaluated the prognosis of T0 stage DTC patients as compared to T1 to T4 stage patients, based on SEER data from patients diagnosed between 2004-2013.

\section{RESULTS}

\section{Demographic and clinical features}

A total of 94092 patients who had definite T stage DTC, according to AJCC versions 6 and 7, were included in this study. The distribution of patients by stage were the following: 180 patients were T0, 55615 were T1, 15613 were T2 stage, 17529 were T3, and 3669 were T4 stage. The study patients' mean age and survival in months for the different $\mathrm{T}$ stages are shown in Table 1 . T0 patients had significantly shorter survival months than patients with other stages.

\section{Cancer specific mortality and all-cause mortality for different stages of DTC}

In the study cohort, the rate of cancer-specific mortality, per 1000 person-years, for T0, T1, T2, T3, and T4 stage were 14.83 (95\% CI, 7.71-28.50), $0.38(95 \%$ CI, 0.31-0.47), 1.11 (95\% CI, 0.89-1.40), 3.40 (95\% CI, 2.99-3.86) and 35.70 (95\% CI, 32.76-38.91), respectively (Table 2). The all-cause mortality, per 1000 person-years, in patients with T0, T1, T2, T3 and T4 stage were 59.31 (95\% CI, 42.78-82.22), 7.68 (95\% CI, 7.32-8.04), 8.52 (95\% CI, 7.85-9.25), 12.58 (95\% CI, 11.78-13.44) and 58.43 (95\% CI, 54.63-62.49).

\section{Risk factors for thyroid cancer-specific mortality and all-cause mortality}

Univariate Cox regression analyses showed that age, male sex, race, TNM stage, follicular subtype, and radiation and surgery approach were significant risk factors of cancer-specific mortality. In the multivariate Cox regression model, T0 stage showed significant higher risk for cancer-specific mortality as compared to $\mathrm{T} 1$ and lower risk for $\mathrm{T} 4$, but not to $\mathrm{T} 2$ and $\mathrm{T} 3$ after adjustment of influential risk factors (Table 3). For the all-cause mortality, univariate Cox regression analyses showed that age, male sex, race, TNM stage, follicular subtype, and radiation and surgery approach were significant risk factors. Multivariate Cox regression analysis determined that T0 stage showed significant lower risk for all-cause mortality compared to T4 stage patients, but not $\mathrm{T} 1-$ T3 stage patients (Table 3 ).

\section{Adjusting for patient characteristics using propensity score matching}

T0 stage patients had a poorer prognosis (both cancer-specific mortality and all-cause mortality) compared to T1-T3 patients. However, they had a better prognosis than T4 stage patients for cancerspecific mortality, and similar with T4 stage patients for all-cause mortality (Figure 1A-1D). To minimize selection bias, propensity scored matching analysis was performed regarding the age, sex, race, N/M stage, histologic subtype, surgery and radiation treatment approaches. In survival analysis, T0 stage had a poorer prognosis for cancer-specific mortality compared to T1 and T2 stage $(\mathrm{p}=0.001,<0.001$, respectively, Figure 2A, 2B), but T0 stage patients had a similar prognosis compared to $\mathrm{T} 3$ stage patients after propensity score matching for age, sex, and race. There was no significant difference between $\mathrm{T} 0$ stage and T1-T4 stages for cancer-specific mortality after propensity score matching for age, sex and race, N/M stage, and histologic subtype (Figure 3A-3D). After matching for all influential factors, including surgery and radiation treatment, T0 stage patients had a worse prognosis for cancer-specific mortality compared to $\mathrm{T} 1$ stage patients $(p=0.001$, Figure $4 \mathrm{~A})$ and better prognosis compared to T4 stage patients $(\mathrm{p}<0.001$, Figure 4D) but not different with T2-T3 stage patients( $p=0.689,0.172$; respectively, Figure 4B, 4C).

In survival analysis for all-cause mortality, T0 stage patients had a poorer prognosis compared to T1-T3 stage patients (all $\mathrm{p}<0.001$, Figure $5 \mathrm{~A}-5 \mathrm{C}$ ), but similar prognosis as T4 stage patients after matching for age, sex and race (Figure 5D). Similar results were obtained after matching for age, sex and race, N/M stage, histologic subtype (Figure 6A-6D). After matching for all influential factors including surgery and radiation treatment, T0 stage patients showed a better prognosis for all-cause mortality compared to T1 stage patients $(\mathrm{p}<0.001$, Figure $7 \mathrm{~A})$, but no difference when compared to T2-T4 patients (Figure 7B-7D).

\section{DISCUSSION}

The previous and current edition (6th and 7th) of the AJCC guidelines defines T0 stage as there being no evidence of a primary tumor. However, the prognosis of T0 stage DTC patients was not thoroughly investigated in the literature. In this study, we evaluated the prognosis of T0 stage DTCs from the SEER database based on diagnoses from 2004-2013. We found that T0 stage 
Table 1: Characteristics for patients with T0-T4

\begin{tabular}{|c|c|c|c|c|c|c|c|}
\hline \multirow[t]{2}{*}{ Covariate } & \multirow[t]{2}{*}{ level } & \multicolumn{5}{|c|}{ T stages } & \multirow[t]{2}{*}{$P$ value } \\
\hline & & T0 $(n=180, \%)$ & T1 $(n=55615, \%)$ & $\mathrm{T} 2(\mathrm{n}=15613, \%)$ & T3 (n=17529,\%) & T4 (n=3669,\%) & \\
\hline Age & & $54.87 \pm 18.73$ & $49.64 \pm 14.37$ & $46.22 \pm 15.94$ & $49.03 \pm 16.41$ & $57.11 \pm 17.65$ & $<0.001$ \\
\hline \multirow[t]{2}{*}{ Sex } & Female & $104(57.8)$ & $45049(81.0)$ & $11721(75.1)$ & $12129(69.2)$ & $2406(65.6)$ & $<0.001$ \\
\hline & Male & $76(42.2)$ & $10566(19.0)$ & 3892 (24.9) & $5400(30.8)$ & $1263(34.4)$ & \\
\hline \multirow[t]{3}{*}{ Race } & White & $146(81.1)$ & $46207(40.0)$ & $12620(80.8)$ & $13888(79.2)$ & $2891(78.8)$ & $<0.001$ \\
\hline & Black & $20(11.1)$ & $3442(6.2)$ & $1152(73.8)$ & $1158(6.6)$ & $192(5.2)$ & \\
\hline & Other & $14(7.8)$ & $5295(9.5)$ & $1639(10.5)$ & $2282(13.0)$ & $563(15.3)$ & \\
\hline Tumor size & & & $9.15 \pm 5.84$ & $29.06 \pm 5.76$ & $35.95 \pm 34.53$ & $36.14 \pm 27.94$ & $<0.001$ \\
\hline \multirow[t]{2}{*}{ N-stage } & No & $30(16.7)$ & $48296(86.8)$ & $12370(79.2)$ & $10479(59.8)$ & $1353(36.9)$ & $<0.001$ \\
\hline & N1 & $132(73.3)$ & $6618(11.9)$ & $2865(18.4)$ & $6529(37.2)$ & $1869(50.9)$ & \\
\hline \multirow[t]{2}{*}{ M-stage } & M0 & $135(75.0)$ & $55445(99.7)$ & $15463(00.0)$ & $17124(97.7)$ & 3187 (86.9) & $<0.001$ \\
\hline & M1 & $45(25.0)$ & $170(0.3)$ & $150(1.0)$ & $405(2.3)$ & $482(13.1)$ & \\
\hline \multirow{2}{*}{$\begin{array}{l}\text { Histologic } \\
\text { subtype }\end{array}$} & Papillary & $159(88.3)$ & $54362(97.7)$ & $13480(86.3)$ & $15825(90.3)$ & $3476(84.7)$ & $<0.001$ \\
\hline & Follicular & $21(11.7)$ & $1250(0.3)$ & $2133(13.7)$ & $1703(9.7)$ & $193(5.3)$ & \\
\hline \multirow[t]{2}{*}{ Multifocality } & No & & $34596(62.2)$ & $9413(60.3)$ & $8776(50.1)$ & $1737(47.3)$ & $<0.001$ \\
\hline & Yes & & $20463(36.3)$ & $5972(38.2)$ & $8360(47.7)$ & $1699(46.3)$ & \\
\hline \multirow[t]{3}{*}{ Radiation } & $\begin{array}{l}\text { None or } \\
\text { refused }\end{array}$ & $89(49.4)$ & $33904(61.0)$ & $5484(35.1)$ & $5017(28.6)$ & $1017(27.7)$ & $<0.001$ \\
\hline & $\begin{array}{l}\text { External } \\
\text { beam } \\
\text { radiation } \\
\text { therapy }\end{array}$ & $11(6.1)$ & $583(1.0)$ & $303(1.9)$ & $433(2.5)$ & $393(10.7)$ & \\
\hline & $\begin{array}{l}\text { Radioactive } \\
\text { I-131 ablation }\end{array}$ & $74(41.1)$ & $20038(36.0)$ & $9451(60.5)$ & $11587(66.1)$ & $2170(59.1)$ & \\
\hline \multirow[t]{4}{*}{ Surgery } & Biopsy & $56(31.1)$ & $565(1.0)$ & $270(17.3)$ & $136(0.8)$ & $232(6.3)$ & $<0.001$ \\
\hline & Lobectomy & $11(6.1)$ & $9994(18.0)$ & $1758(11.2)$ & $1267(7.2)$ & $220(6.0)$ & \\
\hline & $\begin{array}{c}\text { Subtotal or } \\
\text { near-total } \\
\text { thyroidectomy }\end{array}$ & $4(2.2)$ & $2192(39.4)$ & $568(3.6)$ & $503(2.9)$ & $145(4.0)$ & \\
\hline & $\begin{array}{l}\text { Total } \\
\text { thyroidectomy }\end{array}$ & $105(58.3)$ & $42417(76.3)$ & $12869(82.4)$ & $15488(88.4)$ & 3024 (82.4) & \\
\hline Survival months & & $40.47 \pm 30.71$ & $49.00 \pm 33.48$ & $51.05 \pm 34.20$ & $47.77 \pm 33.18$ & $47.63 \pm 34.89$ & $<0.001$ \\
\hline
\end{tabular}

patients had a poorer prognosis than expected after adjustment for influential risk factors.

One of the possible reasons for not finding evidence of a primary tumor, and hence a T0 stage diagnosis, is the tumor is too small for detection [11, 12]. The detection rates of papillary thyroid microcarcinoma
(PTMC) and occult thyroid carcinoma have increased due to the worldwide use of high-resolution sonography and ultrasound-guided fine-needle aspiration biopsy (US-FNAB) $[12,13]$. In particular, US-FNAB has been performed for small thyroid nodules regardless of the nodule size. Kim et al. extracted a PTMC sample with 
Table 2: Hazard ratios of stages for the cancer specific mortality and all cause mortality of DTC

\begin{tabular}{lcccccccc}
\hline T stage & $\begin{array}{c}\text { DTC } \\
\text { mortality, } \\
\text { no. }\end{array}$ & \% & $\begin{array}{c}\text { DTC mortality } \\
\text { per 1,000 } \\
\text { person-years }\end{array}$ & $\mathbf{9 5 \%}$ CI & $\begin{array}{c}\text { All cause } \\
\text { mortality, no. }\end{array}$ & $\begin{array}{c}\text { All cause } \\
\text { mortality per } \\
\mathbf{1 , 0 0 0} \text { person- } \\
\text { years }\end{array}$ \\
\hline T0 & 10 & 5.56 & 14.83 & $7.71-28.50$ & 39 & 21.7 & 59.31 & $42.78-82.22$ \\
T1 & 91 & 0.16 & 0.38 & $0.31-0.47$ & 1811 & 3.26 & 7.68 & $7.32-8.04$ \\
T2 & 77 & 0.49 & 1.11 & $0.89-1.40$ & 576 & 3.69 & 8.52 & $7.85-9.25$ \\
T3 & 243 & 1.38 & 3.40 & $2.99-3.86$ & 891 & 5.08 & 12.58 & $11.78-13.44$ \\
T4 & 556 & 15.2 & 35.70 & $32.76-38.91$ & 905 & 24.67 & 58.43 & $54.63-62.49$ \\
\hline
\end{tabular}

DTC: differential thyroid cancer

Table 3: Risk factors for survival: outcome of differentia thyroid cancer specific mortality and all-cause mortality

\begin{tabular}{|c|c|c|c|c|c|c|c|c|c|}
\hline \multirow[t]{3}{*}{ Covariate } & \multirow[t]{3}{*}{ Level } & \multicolumn{4}{|c|}{ Thyroid cancer specific mortality } & \multicolumn{4}{|c|}{ All cause mortality } \\
\hline & & \multicolumn{2}{|c|}{$\begin{array}{l}\text { Univariate Cox } \\
\text { regression }\end{array}$} & \multicolumn{2}{|c|}{$\begin{array}{c}\text { Multivariate Cox } \\
\text { regression }\end{array}$} & \multicolumn{2}{|c|}{$\begin{array}{l}\text { Univariate } \mathbf{C o x} \\
\text { regression }\end{array}$} & \multicolumn{2}{|c|}{ Multivariate Cox regression } \\
\hline & & $\begin{array}{c}\text { Hazard } \\
\text { Ratio (95\% } \\
\text { CI) }\end{array}$ & p-value & $\begin{array}{c}\text { Hazard } \\
\text { Ratio (95\% } \\
\text { CI) }\end{array}$ & p-value & $\begin{array}{c}\text { Hazard Ratio } \\
(95 \% \text { CI })\end{array}$ & p-value & $\begin{array}{c}\text { Hazard Ratio } \\
(95 \% \text { CI })\end{array}$ & p-value \\
\hline Age & & $\begin{array}{c}1.096(1.091- \\
1.101)\end{array}$ & $<0.001$ & $\begin{array}{c}1.063(1.057- \\
1.068)\end{array}$ & $<0.001$ & $\begin{array}{c}1.086(1.084- \\
1.089)\end{array}$ & $<0.001$ & $1.073(1.070-1.075)$ & $<0.001$ \\
\hline \multirow[t]{2}{*}{ Sex } & Female & ref & & ref & & ref & & ref & \\
\hline & Male & $\begin{array}{c}2.765(2.437- \\
3.137)\end{array}$ & 0.005 & $\begin{array}{c}1.223(1.061- \\
1.409)\end{array}$ & 0.006 & $\begin{array}{c}2.419(2.275- \\
2.572)\end{array}$ & $<0.001$ & $1.598(1.496-1.707)$ & $<0.001$ \\
\hline \multirow[t]{3}{*}{ Race } & White & ref & & ref & & ref & & ref & \\
\hline & Black & $\begin{array}{c}0.993(0.764- \\
1.291)\end{array}$ & 0.958 & $\begin{array}{c}1.144(0.868- \\
1.508)\end{array}$ & 0.340 & $\begin{array}{c}1.267(1.134- \\
1.416)\end{array}$ & $<0.001$ & $1.358(1.208-1.526)$ & $<0.001$ \\
\hline & Other & $\begin{array}{c}1.372(1.114- \\
1.648)\end{array}$ & 0.001 & $\begin{array}{c}0.978(0.798- \\
1.198)\end{array}$ & 0.827 & $\begin{array}{c}0.865(0.778- \\
0.962)\end{array}$ & 0.008 & $0.797(0.711-0.893)$ & $<0.001$ \\
\hline \multirow[t]{5}{*}{ T-stage } & T0 & ref & & ref & & ref & & ref & \\
\hline & $\mathrm{T} 1$ & $\begin{array}{c}0.026(0.013- \\
0.050)\end{array}$ & $<0.001$ & $\begin{array}{c}0.193(0.092- \\
0.404)\end{array}$ & $<0.001$ & $\begin{array}{c}0.125(0.091- \\
0.171)\end{array}$ & $<0.001$ & $0.748(0.521-1.073)$ & 0.115 \\
\hline & $\mathrm{T} 2$ & $\begin{array}{c}0.076(0.039- \\
0.146)\end{array}$ & $<0.001$ & $\begin{array}{c}0.522(0.248- \\
1.097)\end{array}$ & 0.086 & $\begin{array}{c}0.136(0.098- \\
0.188)\end{array}$ & $<0.001$ & $0.873(0.6061 .259)$ & 0.468 \\
\hline & $\mathrm{T} 3$ & $\begin{array}{c}0.118(0.118- \\
0.418)\end{array}$ & $<0.001$ & $\begin{array}{c}1.172(0.572- \\
2.401)\end{array}$ & 0.664 & $\begin{array}{c}0.200(0.145- \\
0.275)\end{array}$ & $<0.001$ & $1.001(0.697-1.437)$ & 0.997 \\
\hline & $\mathrm{T} 4$ & $\begin{array}{c}2.456(1.314- \\
4.590)\end{array}$ & 0.005 & $\begin{array}{c}4.628(2.282- \\
9.389)\end{array}$ & $<0.001$ & $\begin{array}{c}0.970(0.704- \\
1.336)\end{array}$ & 0.850 & $2.223(1.552-3.185)$ & $<0.001$ \\
\hline \multirow[t]{2}{*}{$\mathrm{N}$ stage } & No & ref & & ref & & ref & & ref & \\
\hline & N1 & $\begin{array}{c}4.852(4.245- \\
5.545)\end{array}$ & $<0.001$ & $\begin{array}{c}1.896(1.623- \\
2.216)\end{array}$ & $<0.001$ & $\begin{array}{c}1.626(1.516- \\
1.744)\end{array}$ & $<0.001$ & $1.452(1.336-1.579)$ & $<0.001$ \\
\hline
\end{tabular}

(Continued) 


\begin{tabular}{|c|c|c|c|c|c|c|c|c|c|}
\hline \multirow[t]{2}{*}{ Covariate } & \multirow[t]{2}{*}{ Level } & \multicolumn{4}{|c|}{ Thyroid cancer specific mortality } & \multicolumn{4}{|c|}{ All cause mortality } \\
\hline & & \multicolumn{2}{|c|}{$\begin{array}{l}\text { Univariate Cox } \\
\text { regression }\end{array}$} & \multicolumn{2}{|c|}{$\begin{array}{l}\text { Multivariate Cox } \\
\text { regression }\end{array}$} & \multicolumn{2}{|c|}{$\begin{array}{c}\text { Univariate Cox } \\
\text { regression }\end{array}$} & \multicolumn{2}{|c|}{ Multivariate Cox regression } \\
\hline \multirow[t]{2}{*}{ M-stage } & M0 & ref & & ref & & ref & & ref & \\
\hline & M1 & $\begin{array}{c}48.240 \\
(42.273- \\
55.049)\end{array}$ & $<0.001$ & $\begin{array}{c}5.865(4.958- \\
6.939)\end{array}$ & $<0.001$ & $\begin{array}{c}12.981 \\
(11.826- \\
14.248)\end{array}$ & $<0.001$ & $3.453(3.070-3.885)$ & $<0.001$ \\
\hline \multirow{2}{*}{$\begin{array}{l}\text { Histologic } \\
\text { subtype }\end{array}$} & Papillary & ref & ref & & & ref & & ref & \\
\hline & Follicular & $\begin{array}{c}2.804(2.355- \\
3.339)\end{array}$ & $<0.001$ & $\begin{array}{c}1.494(1.217- \\
1.833)\end{array}$ & $<0.001$ & $\begin{array}{c}1.730(1.565- \\
1.911)\end{array}$ & $<0.001$ & $1.157(1.034-1.294)$ & 0.011 \\
\hline \multirow[t]{3}{*}{ Radiation } & $\begin{array}{l}\text { None or } \\
\text { refused }\end{array}$ & ref & & ref & & ref & & ref & \\
\hline & $\begin{array}{l}\text { Radiation } \\
\text { Beam or } \\
\text { Rdioactive } \\
\text { implants }\end{array}$ & $\begin{array}{c}16.802 \\
(14.194- \\
19.889)\end{array}$ & $<0.001$ & $\begin{array}{c}2.326(1.904- \\
2.841)\end{array}$ & $<0.001$ & $\begin{array}{c}3.773(3.362- \\
4.234)\end{array}$ & $<0.001$ & $1.282(1.119-1.467)$ & $<0.001$ \\
\hline & $\begin{array}{c}\text { Radioisotopes } \\
\text { or Radiation } \\
\text { beam+ } \\
\text { isotopes/ } \\
\text { implants }\end{array}$ & $\begin{array}{c}1.100(0.952- \\
1.271)\end{array}$ & 0.197 & $\begin{array}{l}0.777(0.656- \\
0.919)\end{array}$ & 0.003 & $\begin{array}{l}0.650(0.610- \\
0.694)\end{array}$ & $<0.001$ & $\begin{array}{c}0.0683(0.634- \\
0.736)\end{array}$ & $<0.001$ \\
\hline \multirow[t]{4}{*}{ Surgery } & Biopsy & ref & & ref & & ref & & ref & \\
\hline & Lobectomy & $\begin{array}{l}0.031(0.024- \\
0.041)\end{array}$ & $<0.001$ & $\begin{array}{l}0.480(0.352- \\
0.653)\end{array}$ & $<0.001$ & $\begin{array}{l}0.081(0.072- \\
0.092)\end{array}$ & $<0.001$ & $0.317(0.275-0.365)$ & $<0.001$ \\
\hline & $\begin{array}{c}\text { Subtotal or } \\
\text { near-total } \\
\text { thyroidectomy }\end{array}$ & $\begin{array}{l}0.067(0.049- \\
0.091)\end{array}$ & $<0.001$ & $\begin{array}{c}0.553(0.382- \\
0.802)\end{array}$ & 0.002 & $\begin{array}{c}0.088(0.074- \\
0.103)\end{array}$ & $<0.001$ & $0.323(0.269-0.388)$ & $<0.001$ \\
\hline & $\begin{array}{c}\text { Total } \\
\text { thyroidectomy }\end{array}$ & $\begin{array}{c}0.044(0.038- \\
0.053)\end{array}$ & $<0.001$ & $\begin{array}{c}0.444(0.352- \\
0.559)\end{array}$ & $<0.001$ & $\begin{array}{c}0.067(0.061- \\
0.074)\end{array}$ & $<0.001$ & $0.297(0.262-0.336)$ & $<0.001$ \\
\hline
\end{tabular}

a $1 \mathrm{~mm}$ tumor size by US-FNAB [12]. Therefore, the primary tumor may not be detected when undergoing a thyroidectomy.

The balance between inadequate and excessive treatment is a pivotal concern in the management of thyroid carcinoma. Currently, no local surgery or radiation treatment being recommended for T0 stage patients may a consequence of the 2015 ATA guidelines for the management of thyroid nodules and DTC [6]. In addition, surgeons and oncologists choosing to the current management course for T0 patients may be due to the observed low-mortality rate $[7,10]$.

Postoperative radioiodine ablation treatment can eradicate normal-thyroid remnants to reduce or eliminate serum thyroglobulin levels, as well as irradiate neoplastic foci, thereby decreasing the risk of mortality and recurrence [14]. However, radioiodine may also induce lacrimal and salivary gland toxicities $[15,16]$. At present, few studies have examined the administration of radioiodine to provide therapeutic benefits after a complete thyroidectomy. In our current study, only $41.1 \%$ of patients with T0 stage underwent radioactive I-131 ablation as compared with $60.5 \%$ with T2 stage, $66.1 \%$ with T3 stage, and $59.1 \%$ with T4 stage, according to the SEER database.

Nevertheless, our study demonstrated that 58.3\% of patients underwent total thyroidectomy as compared to $76.3 \%$ with T1 stage, $82.4 \%$ with T2 stage, $88.4 \%$ with T3 stage, and $82.4 \%$ with T4 stage. Therefore, a conservative treatment approach may play an important role in DTCs' mortality with T0 stage patients.

Other clinicopathological features, such as more aggressive tumor histologies, multifocality, lymph node metastasis, and extrathyroidal extension, may play a larger role than tumor size alone with regard to patient prognosis [17-20]. In this study, for example, lymph node metastasis accounts for $73.3 \%$ of T0 stage patients, but $11.9 \%$ of T1 stage patients had lymph node metastasis.

T0 patients had a higher incidence of distant metastasis $(45 / 180,25.0 \%)$ than any other $\mathrm{T}$ stages 

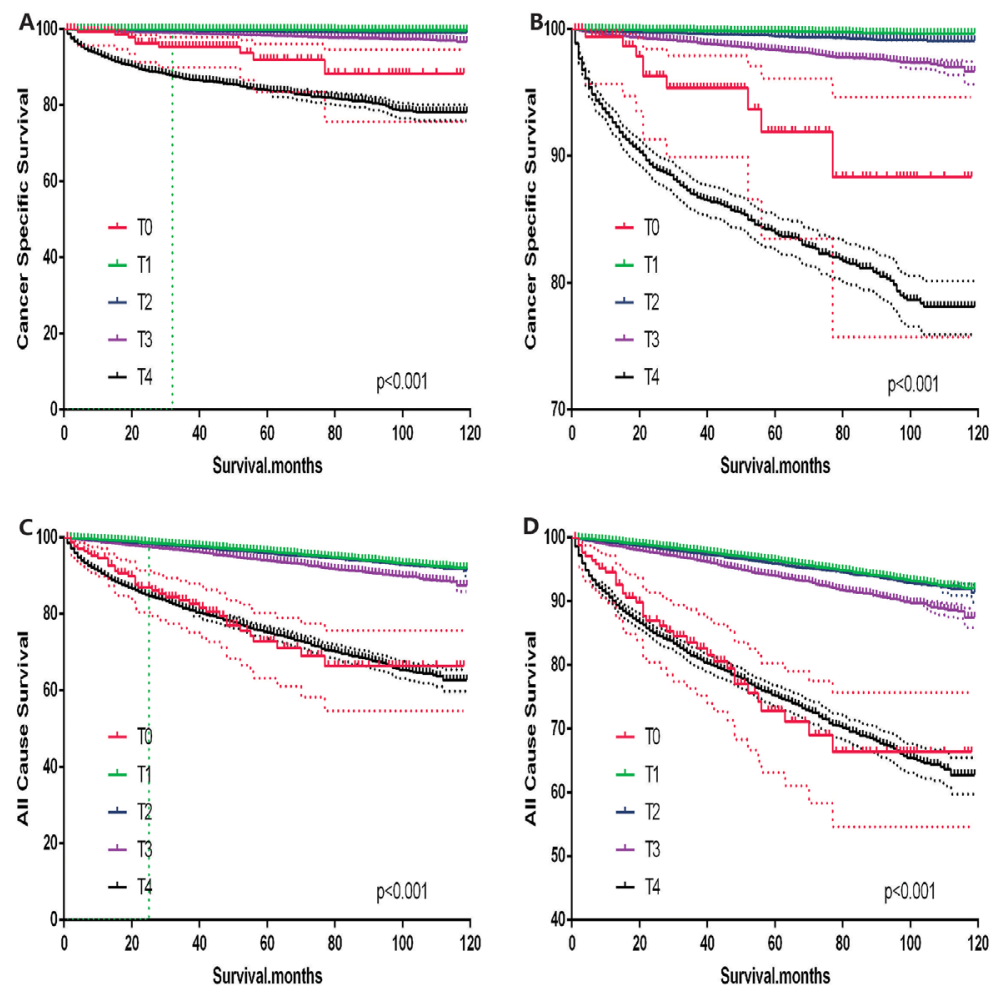

Figure 1: Kaplan Meier curves among patients stratified by T-stage for cancer-specific mortality (A, B) Log rank test $\mathrm{p}<$ $0.0001)$ and all cause mortality $(\mathbf{C}, \mathbf{D}) \log$ rank test $\mathrm{p}<0.0001)$.
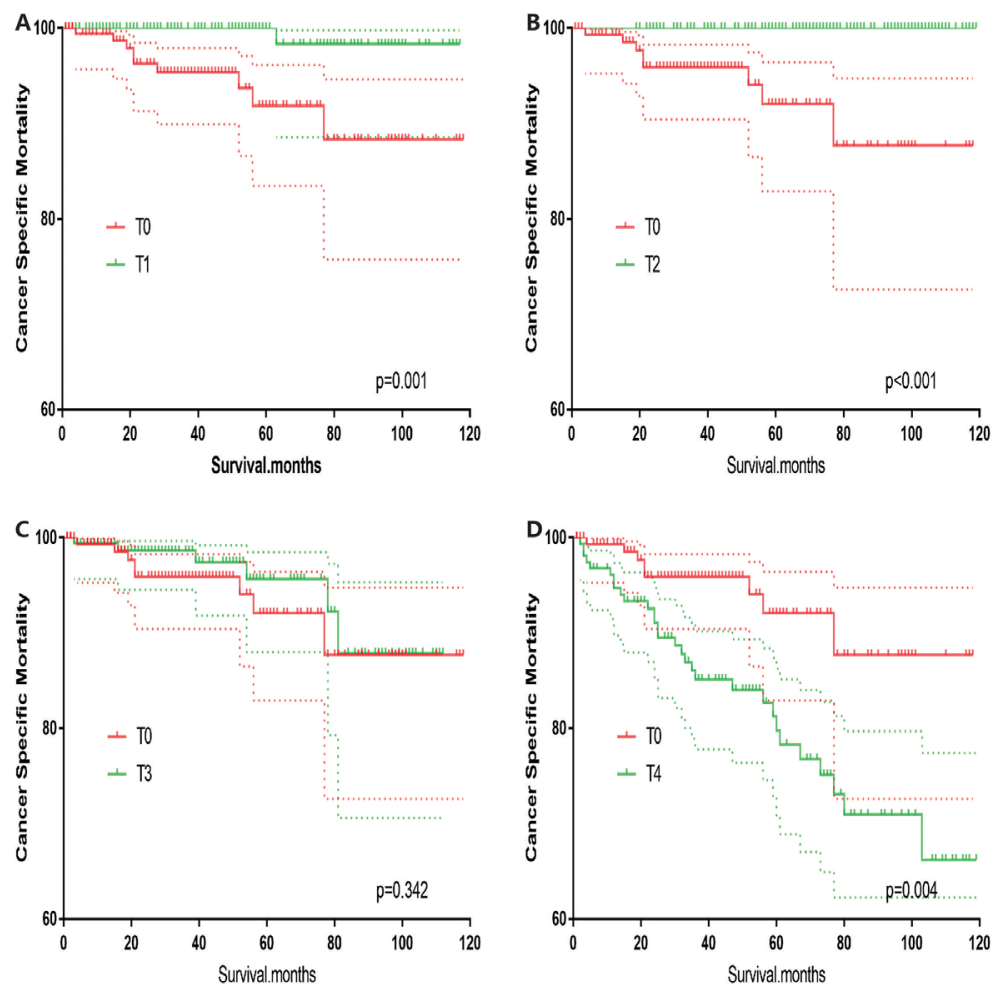

Figure 2: Kaplan Meier curves of cancer-specific mortality for matched T-stage pairs. Age, sex and race matching between $\mathrm{T} 0$ and T1 (A), T0 and T2 (B), T0 and T3 (C), T0 and T4 (D) patients. 

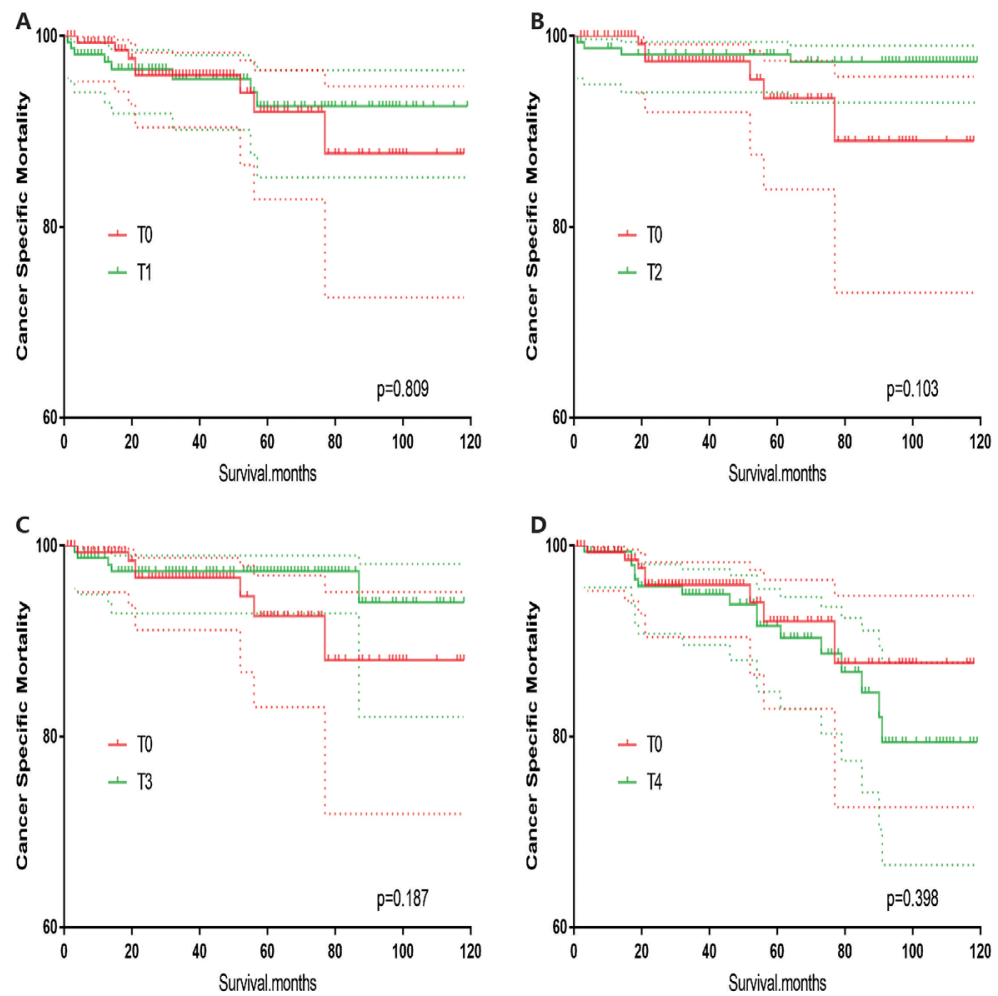

Figure 3: Kaplan Meier curves of cancer-specific mortality for matched T-stage pairs. Age, sex, race, N/M stage, histologic subtype matched between T0 and T1 (A), T0 and T2 (B), T0 and T3 (C), T0 and T4 (D) patients.
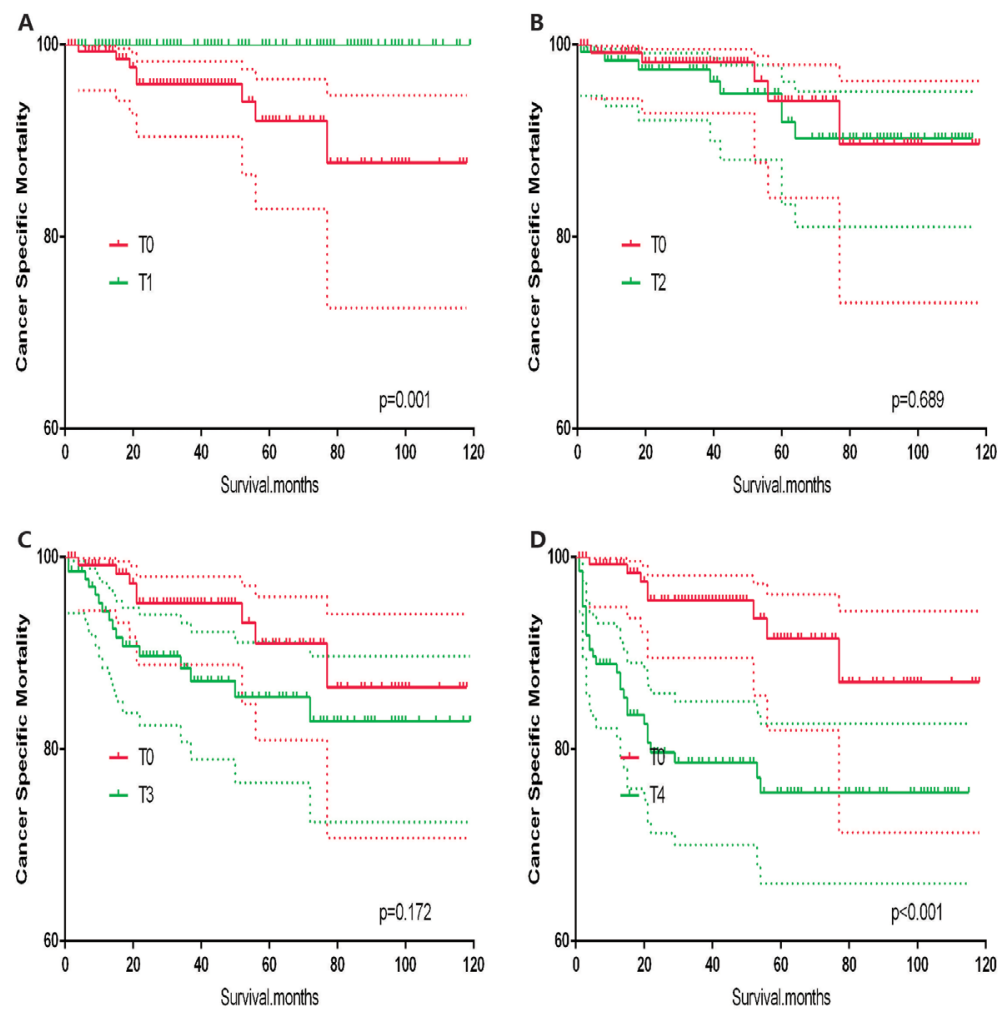

Figure 4: Kaplan Meier curves of cancer-specific mortality for matched T-stage pairs. Age, sex, race, N/M stage, histologic subtype, surgery and radiation treatment matched between T0 and T1 (A), T0 and T2 (B), T0 and T3 (C), T0 and T4 (D) patients. 

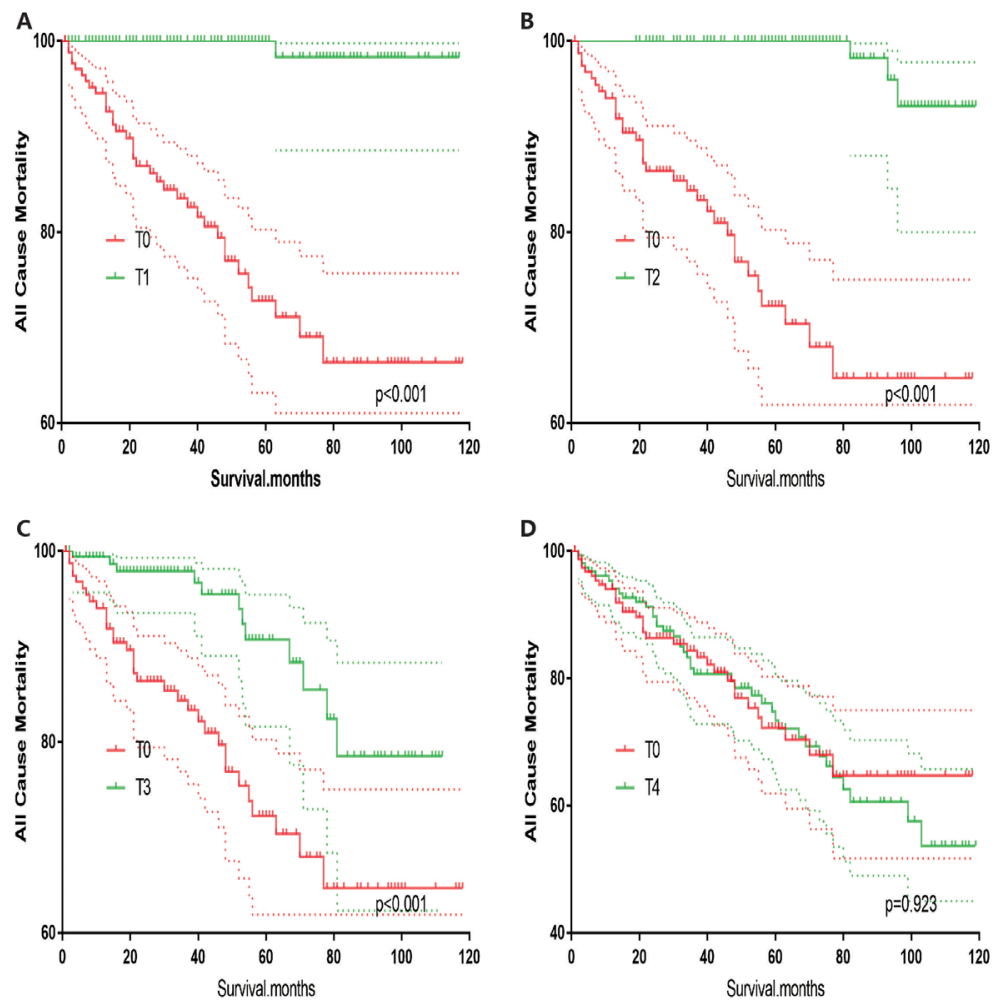

Figure 5: Kaplan Meier curves of all cause mortality for matched T-stage pairs. Age, sex and race matching between T0 and T1 (A), T0 and T2 (B), T0 and T3 (C), T0 and T4 (D) patients.
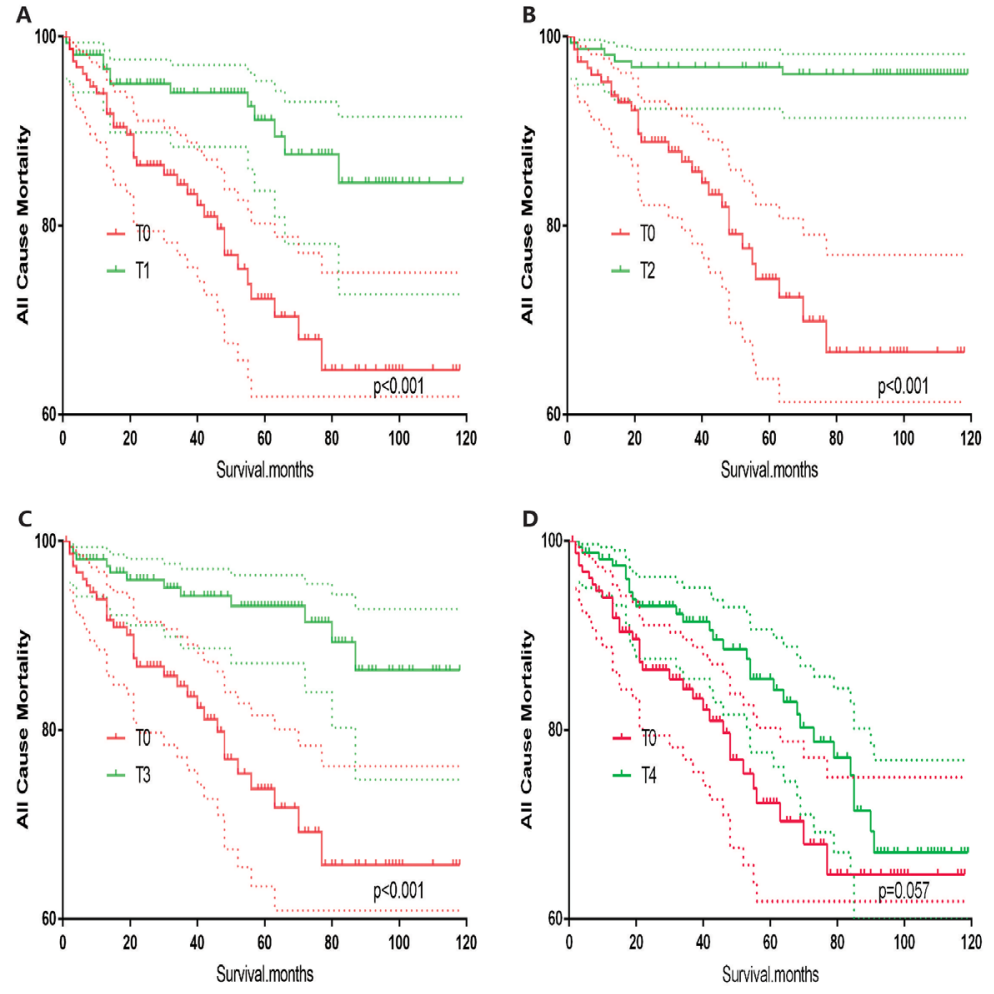

Figure 6: Kaplan Meier curves of all cause mortality for matched T-stage pairs. Age, sex, race, N/M stage, histologic subtype matching between T0 and T1 (A), T0 and T2 (B), T0 and T3 (C), T0 and T4 (D) patients. 
patients did in this study. According to previous studies, distant metastasis was a significant risk for thyroid cancer-specific mortality and all-cause mortality [17, 21]. Therefore, the high prevalence of distant metastasis in T0 stage may result in a higher mortality from DTC. Our hypothesis was also strengthened by the results from the propensity score matching analysis.

Our study had the following limitations. One limitation of this study is that the utilized dataset lacked information regarding recurrence, thereby introducing overestimation bias when designating cancer-specific
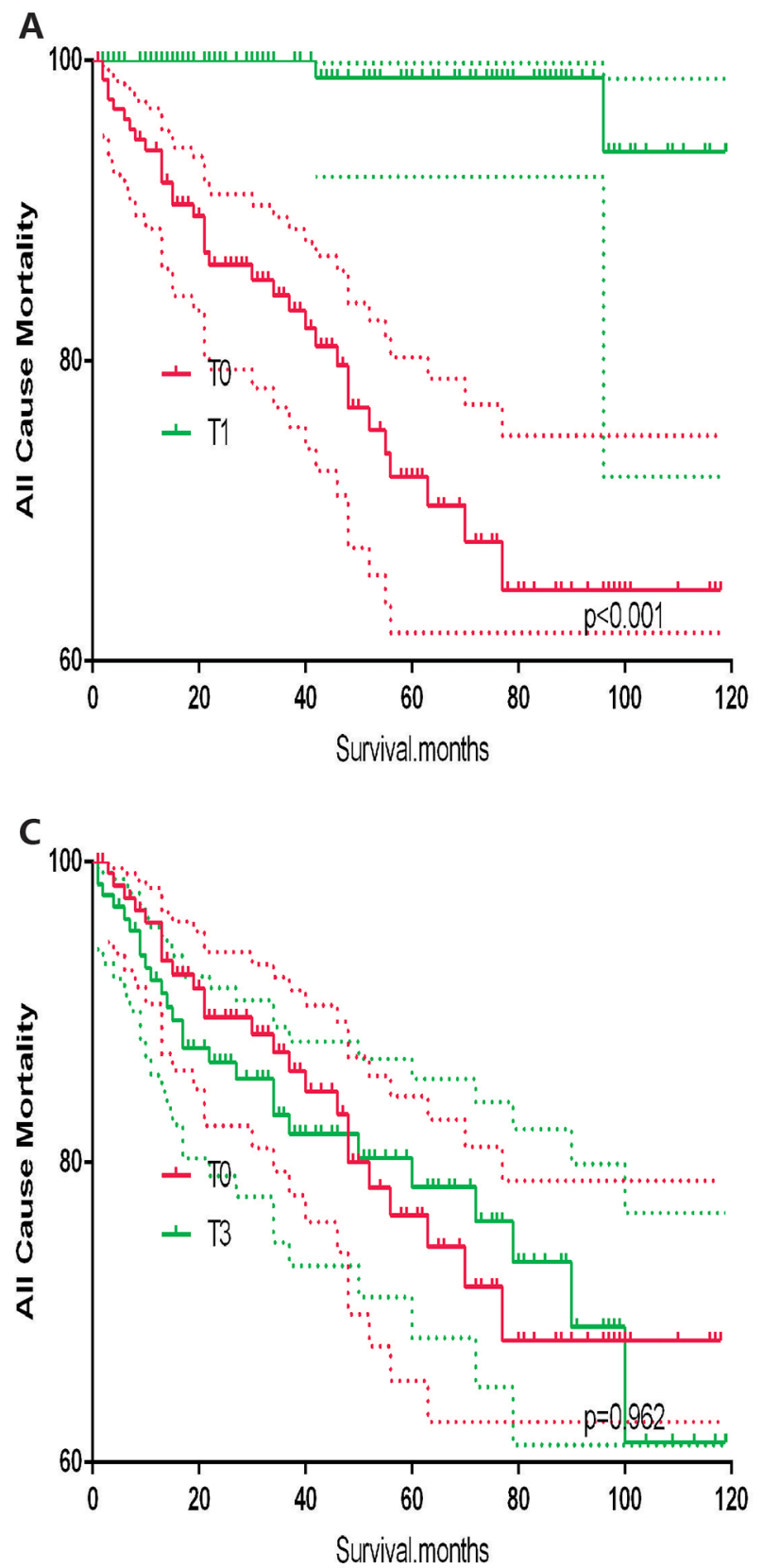

death and all-cause death. Another limitation of this study is that family history, vascular invasion, and other histologic findings were not evaluated or included in our study. Furthermore, the molecular markers such as $B R A F$ point mutation and TERT promoter point mutations were not observed in our study or adjusted for in our analyses.

In summary, T0 patients had a significantly poorer survival than T1-T3 patients. These results are not consistent with current expectations of DTC progression and raise new implications for the treatment of patients with T0 stage DTC.
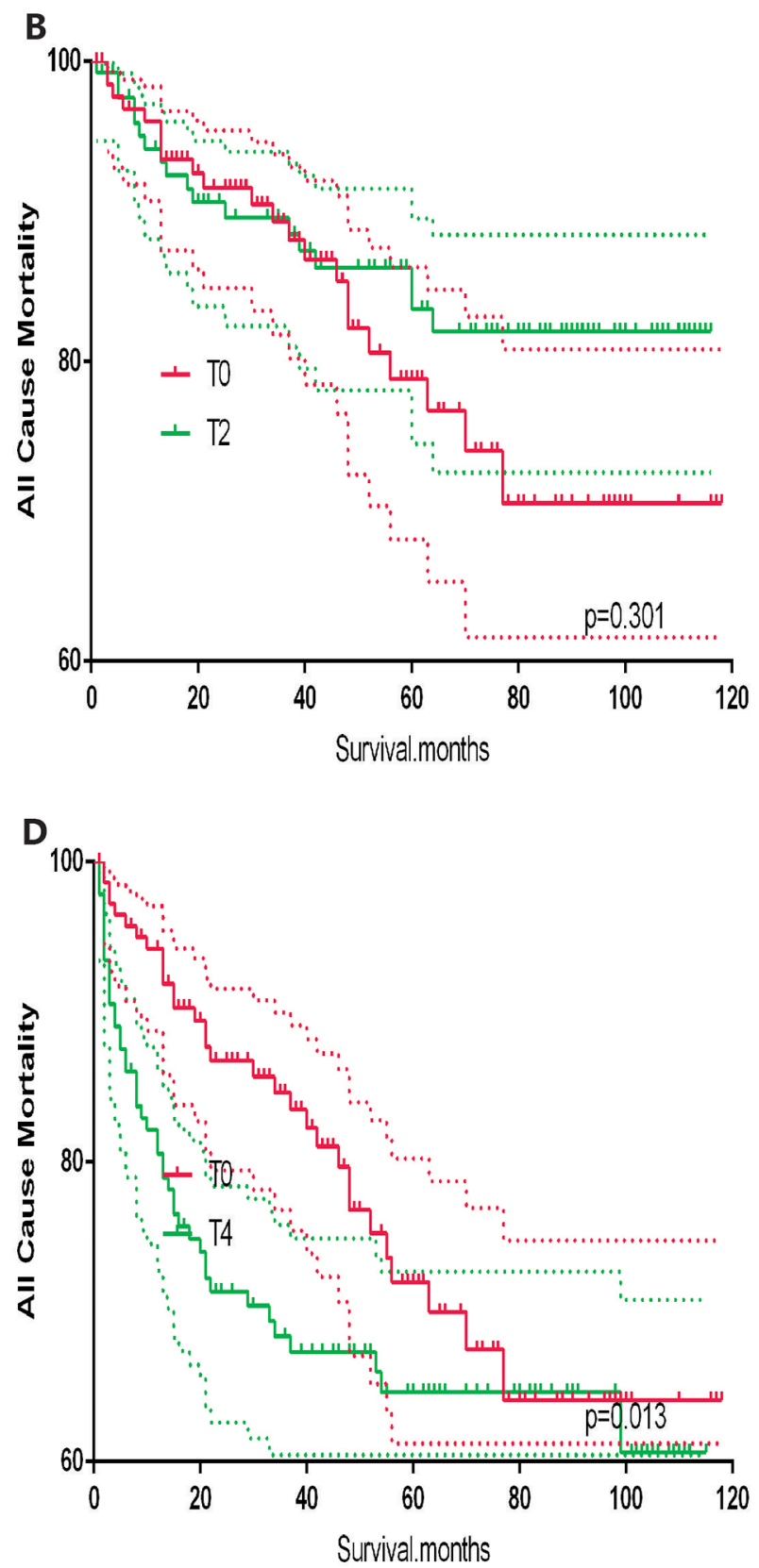

Figure 7: Kaplan Meier curves of all cause mortality for matched T-stage pairs. Age, sex, race, N/M stage, histologic subtype, surgery and radiation treatment matching between T0 and T1 (A), T0 and T2 (B), T0 and T3 (C), T0 and T4 (D) patients. 


\section{MATERIALS AND METHODS}

\section{Study population}

We investigated a large number of DTC patients from the SEER program. The SEER project is a United States population-based cancer registry that began in 1973, and is supported by both the National Cancer Institute and Centers for Disease Control and Prevention. It contains cancer data from across multiple geographic regions on the incidence, prevalence, mortality, population-based variables, primary tumor characteristics, and more.

\section{Data collection and analysis}

We examined SEER data from 2004 to 2013 and selected patients with a diagnosis of DTC, as defined by a combination of ICD-O site code of C73.9 (i.e., thyroid, papillary, and/or follicular histology). The diagnosis codes were included in the study: "papillary carcinoma", "papillary adenocarcinoma", "oxyphilic adenocarcinoma", "follicular adenocarcinoma", "papillary \& follicular adenocarcinoma", and "papillary cyst-adenocarcinoma". To compare the survival rate among different $\mathrm{T}$ stages, 94092 patients were categorized according to AJCC T staging (version 6 and 7, Supplementary Tables 1 and 2). Age, sex, race, N/M stage, histologic subtype, surgery (biopsy, lobectomy, subtotal or near-total thyroidectomy, and total thyroidectomy) and radiation (none or refused, external beam radiation therapy, and radioactive I-131 ablation) treatments were evaluated in patients with different $\mathrm{T}$ stages.

\section{Statistical analyses}

Patients were followed-up until December 2013. Patient survival curves (thyroid cancer-specific mortality and all-cause mortality) were examined by KaplanMeier analyses with the log-rank test. To further adjust for potential baseline confounding factors, a propensity score matching analysis was conducted. Cox proportional hazards regression analyses were performed to estimate the hazard ratios with $95 \%$ Cis, to show the magnitude of the effect of stage on cancer-specific mortality and all-cause mortality [22]. All p-values were 2-sided, with $\mathrm{p}<.05$ being considered significant. Analyses were performed using SPSS version 19.0, Stata/SE version 12 (Stata Corp.), and GraphPad Prism version 6 (GraphPad Software Inc.).

\section{Author contributions}

All authors contributed to the design of the study and writing of the manuscript C. P. L and J.M undertook the research and performed the analyses. All authors reviewed and approved the final version of the manuscript.

\section{ACKNOWLEDGMENTS}

None.

\section{CONFLICTS OF INTEREST}

The authors declare no conflicts of interest.

\section{FUNDING}

None.

\section{REFERENCES}

1. Mao Y, Xing M. Recent incidences and differential trends of thyroid cancer in the USA. Endocr Relat Cancer. 2016; 23:313-322.

2. Siegel R, DeSantis C, Virgo K, Stein K, Mariotto A, Smith T, Cooper D, Gansler T, Lerro C, Fedewa S, Lin C, Leach C, Cannady RS, et al. Cancer treatment and survivorship statistics, 2012. CA Cancer J Clin. 2012; 62:220-241.

3. Chen W, Zheng R, Baade PD, Zhang S, Zeng H, Bray F, Jemal A, Yu XQ, He J. Cancer statistics in China, 2015. CA Cancer J Clin. 2016; 66:115-132.

4. Xing M. Molecular pathogenesis and mechanisms of thyroid cancer. Nat Rev Cancer. 2013; 13:184-199.

5. Fagin JA, Wells SA Jr. Biologic and clinical perspectives on thyroid cancer. N Engl J Med. 2016; 375:1054-1067.

6. Haugen BR, Alexander EK, Bible KC, Doherty GM, Mandel SJ, Nikiforov YE, Pacini F, Randolph GW, Sawka AM, Schlumberger M, Schuff KG, Sherman SI, Sosa JA, et al. 2015 American Thyroid Association Management Guidelines for adult patients with thyroid nodules and differentiated thyroid cancer: the American Thyroid Association Guidelines Task Force on thyroid nodules and differentiated thyroid cancer. Thyroid. 2016; 26:1-133.

7. Zhou P, Tian S, Li J, Zhao Y, Liu W, Zhang Y, Hu Z. Paradoxes in thyroid carcinoma treatment: analysis of the SEER database 2010-2013. Oncotarget. 2017; 8:345-353. https://doi.org/10.18632/oncotarget.13395.

8. Lim H, Devesa SS, Sosa JA, Check D, Kitahara CM. Trends in thyroid cancer incidence and mortality in the United States, 1974-2013. JAMA. 2017; 317:1338-1348.

9. Adam MA, Thomas S, Hyslop T, Scheri RP, Roman SA, Sosa JA. Exploring the relationship between patient age and cancer-specific survival in papillary thyroid cancer: rethinking current staging systems. J Clin Oncol. 2016; 34:4415-4420.

10. Sarda AK, Aggarwal S, Pandey D, Gautam G. Prognostic factors for well-differentiated thyroid cancer in an endemic area. Asian J Surg. 2002; 25:325-329.

11. Alecu L, Barbulescu M, Ursut B, Enciu O, Slavu I, Braga V. Occult thyroid carcinoma in our experience -- should 
we reconsider total thyroidectomy for benign thyroid pathology? Chirurgia (Bucur). 2014; 109:191-197.

12. Kim DW, Kim SH, Jung SJ. Successful sonography-guided fine-needle aspiration biopsy of a 1-millimeter-diameter papillary thyroid microcarcinoma. AJNR Am J Neuroradiol. 2010; 31:1082-1084.

13. Liu Z, Zeng W, Liu C, Wang S, Xiong Y, Guo Y, Li X, Sun S, Chen T, Maimaiti Y, Yu P, Huang T. Diagnostic accuracy of ultrasonographic features for lymph node metastasis in papillary thyroid microcarcinoma: a singlecenter retrospective study. World J Surg Oncol. 2017; 15:32.

14. Schlumberger M, Catargi B, Borget I, Deandreis D, Zerdoud S, Bridji B, Bardet S, Leenhardt L, Bastie D, Schvartz C, Vera P, Morel O, Benisvy D, et al. Strategies of radioiodine ablation in patients with low-risk thyroid cancer. N Engl J Med. 2012; 366:1663-1673.

15. Kloos RT, Duvuuri V, Jhiang SM, Cahill KV, Foster JA, Burns JA. Nasolacrimal drainage system obstruction from radioactive iodine therapy for thyroid carcinoma. J Clin Endocrinol Metab. 2002; 87:5817-5820.

16. Mandel SJ, Mandel L. Radioactive iodine and the salivary glands. Thyroid. 2003; 13:265-271.

17. Liu Z, Wang L, Yi P, Wang CY, Huang T. Risk factors for central lymph node metastasis of patients with papillary thyroid microcarcinoma: a meta-analysis. Int J Clin Exp Pathol. 2014; 7:932-937.

18. Pisanu A, Reccia I, Nardello O, Uccheddu A. Risk factors for nodal metastasis and recurrence among patients with papillary thyroid microcarcinoma: differences in clinical relevance between nonincidental and incidental tumors. World J Surg. 2009; 33:460-468.

19. Liu Z, Zeng W, Chen T, Guo Y, Zhang C, Liu C, Huang T. A comparison of the clinicopathological features and prognoses of the classical and the tall cell variant of papillary thyroid cancer: a meta-analysis. Oncotarget. 2017; 8:6222-6232. https://doi.org/10.18632/oncotarget.14055.

20. Rossi R, Roti E, Trasforini G, Pansini G, Cavazzini L, Zatelli MC, Pearce EN, Braverman LE, Uberti EC. Differentiated thyroid cancers $11-20 \mathrm{~mm}$ in diameter have clinical and histopathologic characteristics suggesting higher aggressiveness than those $<$ or $=10 \mathrm{~mm}$. Thyroid. 2008; 18:309-315.

21. Lin JD, Hsueh C, Chao TC. Long-term follow-up of the therapeutic outcomes for papillary thyroid carcinoma with distant metastasis. Medicine. 2015; 94:e1063.

22. Spruance SL, Reid JE, Grace M, Samore M. Hazard ratio in clinical trials. Antimicrob Agents Chemother. 2004; 48:2787-2792. 